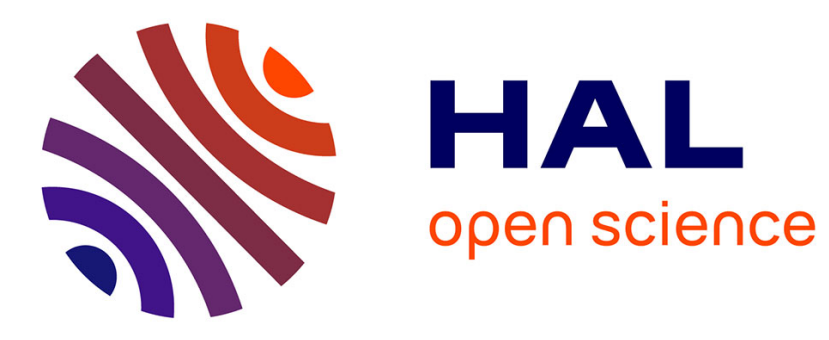

\title{
Magnetic particle mixing with magnetic micro-convection for microfluidics
}

Guntars Kitenbergs, Kaspars Erglis, Régine Perzynski, Andrejs Cēbers

\section{To cite this version:}

Guntars Kitenbergs, Kaspars Erglis, Régine Perzynski, Andrejs Cēbers. Magnetic particle mixing with magnetic micro-convection for microfluidics. Journal of Magnetism and Magnetic Materials, 2015, 380, pp.227 - 230. 10.1016/j.jmmm.2014.10.033 . hal-01117158

\section{HAL Id: hal-01117158 https://hal.sorbonne-universite.fr/hal-01117158}

Submitted on 16 Feb 2015

HAL is a multi-disciplinary open access archive for the deposit and dissemination of scientific research documents, whether they are published or not. The documents may come from teaching and research institutions in France or abroad, or from public or private research centers.
L'archive ouverte pluridisciplinaire HAL, est destinée au dépôt et à la diffusion de documents scientifiques de niveau recherche, publiés ou non, émanant des établissements d'enseignement et de recherche français ou étrangers, des laboratoires publics ou privés. 


\title{
Magnetic particle mixing with magnetic micro-convection for microfluidics
}

\author{
Guntars Kitenbergs* ${ }^{* 1,2,3}$, Kaspars Ërglis ${ }^{1}$, Régine Perzynski ${ }^{2}, 3$, and \\ Andrejs Cēbers ${ }^{1}$ \\ ${ }^{1}$ MMML lab, Department of Theoretical Physics, University of Latvia, \\ Zellu 8, LV-1002, Rīga, Latvia \\ ${ }^{2}$ Sorbonne Universités, UPMC Univ Paris 06, UMR 8234, PHENIX, \\ 4 place Jussieu, F-75005, Paris, France \\ ${ }^{3}$ CNRS, UMR 8234, PHENIX, 4 place Jussieu, F-75005, Paris, France
}

\section{Paper}

This paper has been published to Journal of Magnetism and Magnetic Materials, (2015), 380, 227-230. doi:10.1016/j.jmmm.2014.10.033.

\begin{abstract}
In this paper we discuss the magnetic micro-convection phenomenon as a tool for mixing enhancement in microfluidics systems in cases when one of the miscible fluids is a magnetic particle colloid. A system of a water-based magnetic fluid and water is investigated experimentally under homogeneous magnetic field in a Hele-Shaw cell. Subsequent image analysis both qualitatively and quantitatively reveals the high enhancement of mixing efficiency provided by this method. The mixing efficiency dependence on the magnetic field and the physical limits is discussed. A suitable model for a continuous-flow microfluidics setup for mixing with magnetic micro-convection is also proposed and justified with an experiment. In addition, possible applications in improving the speed of ferrohydrodynamic sorting and magnetic label or selected tracer mixing in lab on a chip systems are noted.
\end{abstract}

\footnotetext{
*Email address for correspondence: guntars.kitenbergs@lu.lv
} 


\section{Keywords}

Microfluidics, Mixing, Magnetic Fluid, Ferrofluid, Magnetic micro-convection, Diffusion

\section{Introduction}

Over the last 15 years the field of microfluidics has continuously advanced, providing an interesting framework for various applications and scientific studies. As these systems typically have a small Reynolds number, a lot of effort has been devoted to enhance mixing, which is otherwise limited by diffusion speed [1, causing long channel lengths. Mixers in microfluidics can be divided into passive and active, where the latter need an external energy supply [2]. A convenient energy source for active mixing systems is an external magnetic field, as the energy can be transmitted to the microfluidics chip or cell without direct connectors. It is particularly interesting for systems with magnetic particles. Magnetic fluid, being a colloidal disperion of magnetic particles, can be used as a model system for future applications. Several examples of magnetic and non-magnetic fluid mixing have already been demonstrated [3, 4]. Here we evaluate the possible use of the magnetic micro-convection phenomenon for mixing applications, applying the knowledge obtained in previous theoretical and experimental studies [5, 6].

\section{Theory, Materials and Methods}

Magnetic micro-convection, first described in 1980s [7, is caused by a ponderomotive force of the non-homogeneous self magnetic field of the magnetic fluid. Above a certain magnetic field threshold, an instability forms on the magnetic/non-magnetic fluid interface. A characteristic fingering pattern results from the magnetic particle flow induced by the acting force, pushing magnetic particles into the non-magnetic liquid. This force depends on particle concentration gradient, which decreases with mixing time as a result of the ongoing particle diffusion. Here we will focus on the practical application of the magnetic micro-convection, whereas more information on the fundamental aspects can be found in our previous studies [5, 6].

Experimentally magnetic micro-convection is tested in a Hele-Shaw cell as a microfluidics model system. It is placed on a stage with a coil system in an inverted microscope (Leica DMI3000B) with a 10x magnification (See Fig 1). The coil system (Fig 1 (1)) consists of two identical coils that are $19 \mathrm{~mm}$ high and have inner and outer diameters of $d_{\text {in }}=45 \mathrm{~mm}$ and $d_{\text {out }}=57 \mathrm{~mm}$ respectively. Each coil has 200 turns of copper wire with a diameter $d=0.7 \mathrm{~mm}$. Coils are fixed on both sides of a microscope stage (1 mm thick, Fig 1 (2)) so that their axes and the optical axis of the microscope coincide. A current (up to 3 A) flowing through the coils that are connected in series $\left(R_{t o t}=2.9 \Omega\right)$ creates a $\approx 25 \mathrm{~mm}^{2}$ large area with a homogeneous magnetic field in $z$ direction (up to 


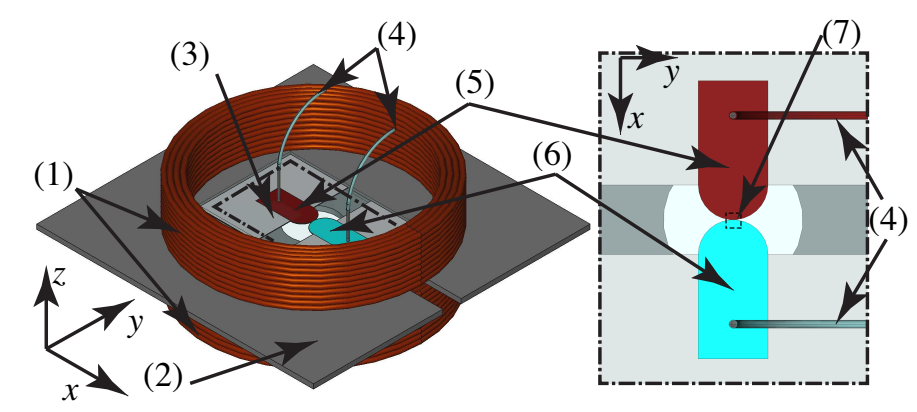

Figure 1: Experimental setup consists of a coil system (1) fitted on a microscope stage (2). A Hele-Shaw cell (3) with tubing connections for fluid introduction (4) is placed in the center. A closer view of the cell displays how magnetic fluid (5) and water (6) droplets are brought to a contact in the center of the Hele-Shaw cell. Camera is recording only the central part of the cell (7) where droplets merge.

$B=20 \mathrm{mT}$ ) where the center of the cell is placed. The cell (Fig 1 (3)) is made of two Parafilm M@ spacers, cut in 'U' shapes, that are put opposite each other between two glass slides to form a rectangular cell leaving two outlets for air on the sides. Two metal tubes, glued in drilled holes in the upper glass slide, provide tubing connections (Fig 1 (4)) to a syringe pump. After assembly, glass slides with spacers are welded together on a hot plate $\left(75^{\circ} \mathrm{C}, 5 \mathrm{~min}\right)$, creating a cell with a $5 \times 20 \times 0.12 \mathrm{~mm}^{3}$ size. The process is filmed in the bright-field mode with a fast camera (Mikrotron MC1363, maximum resolution $1280 \times 1024 \mathrm{px}$ ). It is recording true color images at $f=50 \mathrm{~Hz}$ and its white balance is calibrated for the tungsten halogen lamp used for illumination. Color images are later converted to 8-bit grayscale images $\left(I_{\mathrm{GRAY}}=0.2989 \times I_{\mathrm{R}}+0.5870 \times I_{\mathrm{G}}+\right.$ $\left.0.1140 \times I_{\mathrm{B}}\right)$ for subsequent analysis.

The miscible fluids are water based magnetic fluid (Fig 1 (5)) and distilled water (Fig 1 (6)). The magnetic fluid is made by Massart's coprecipitation method [8] and results in maghemite particles that are stabilized with citrate ions and have a mean diameter $d=7.0 \mathrm{~nm}$, saturation magnetization $M_{\text {sat }}=$ $8.4 \mathrm{kA} / \mathrm{m}$ at $B_{\text {sat }}=1 \mathrm{~T}$, susceptibility $\chi_{0}=0.2$ (SI units) in the range up to $B=20 \mathrm{mT}$ and volume fraction $\phi=2.9 \%$ (from magnetization measurements).

Both fluids are slowly brought into contact inside the cell through the tubing connections by a syringe pump, while the magnetic field is already present. When droplets touch and an interface is formed along $y$ axis, the pump is turned off, stopping the flow of fluids. The magnetic micro-convection is recorded for further analysis in $0.5 \times 0.5 \mathrm{~mm}^{2}$ area (Fig $1(7)$ ) with the microscope camera, forming images $I_{t}(i, j)$, where $t$ is time, and $i$ and $j$ denote spatial indices with the total length $N=360 \mathrm{px}$ for both $x$ and $y$ axes (see Fig2 2 (a)).

To characterize a mixing system quantitatively, information on concentration distribution is necessary. As we use bright field microscopy and magnetic fluid 

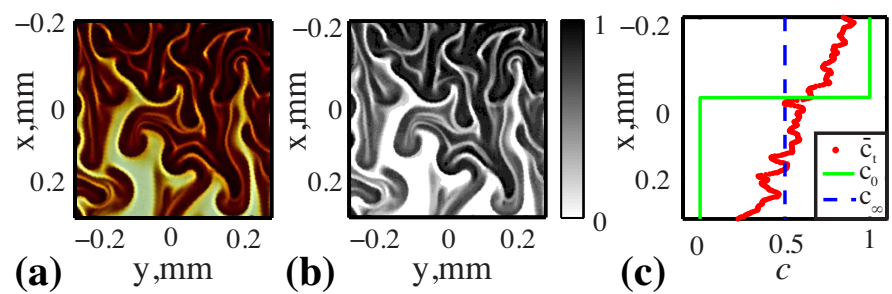

Figure 2: Example of image analysis sequence for finding concentration distribution. (a) Original image $I$. (b) Concentration plot $c$, found via BeerLambert law (Eq11). (c) Spatially averaged concentration $\bar{c}$ calculated with $\mathrm{Eq} 2$ is marked with dots, while theoretical initial state $c_{0}$ and final mixed state $c_{\infty}$ are marked with solid and dashed lines.

is absorbing light, we find normalized concentration plots $c_{t}(i, j)$ from images $I_{t}(i, j)$ via Beer-Lambert law (see Fig $2(\mathrm{~b})$ ):

$$
c_{t}(i, j)=\frac{\lg I_{t}(i, j)-\lg I_{\mathrm{H}_{2} \mathrm{O}}}{\lg I_{\mathrm{FF}}-\lg I_{\mathrm{H}_{2} \mathrm{O}}},
$$

where $I_{\mathrm{H}_{2} \mathrm{O}}$ and $I_{\mathrm{FF}}$ are the intensities of initial water and magnetic fluid concentrations, found in images at $t=0$. A spatially averaged concentration $\bar{c}_{t}(i)$ is then calculated from concentration data, to characterize concentration in the mixing direction along $x$ axis (see Fig 2 (c)):

$$
\bar{c}_{t}(i)=\frac{1}{N} \sum_{j=1}^{N} c_{t}(i, j) .
$$

To quantify mixing dynamics, we define mixing efficiency $M_{\text {eff }}(t)$ as follows:

$$
M_{\mathrm{eff}}(t)=1-\frac{\sqrt{\frac{1}{N} \sum_{i=1}^{N}\left(\bar{c}_{t}(i)-c_{\infty}(i)\right)^{2}}}{\sqrt{\frac{1}{N} \sum_{i=1}^{N}\left(c_{0}(i)-c_{\infty}(i)\right)^{2}}},
$$

where $c_{0}$ and $c_{\infty}$ are theoretical concentration distributions before mixing starts $(t=0)$ and when mixing has finished $(t \rightarrow \infty)$ (see Fig[2(c)). Definition of $M_{\text {eff }}$ is a slight variation of other measurements from literature, e.g. mixing ratio [3] and percentage mixed [9], adjusted for better representation of the experimental data.

The experiment involves an interface formation, which creates a slightly mixed state that differs from one time to another, due to the experimental limitations instead of a theoretical step like concentration distribution $c_{0}$. We remove this influence by introducing a relative mixing efficiency $M_{\mathrm{r}}(t)$ for $t>t_{0}$, which subtracts the mixing efficiency that has been made due to the interface 
formation. This value $M_{\text {eff }}\left(t_{0}\right)$ is taken at a manually chosen time $t_{0}$, when it can be seen that the interface formation is finished (typically $t_{0}=0.04 \mathrm{~s}$ ):

$$
M_{\mathrm{r}}(t)=M_{\mathrm{eff}}(t)-M_{\mathrm{eff}}\left(t_{0}\right) .
$$

\section{Results and Discussion}
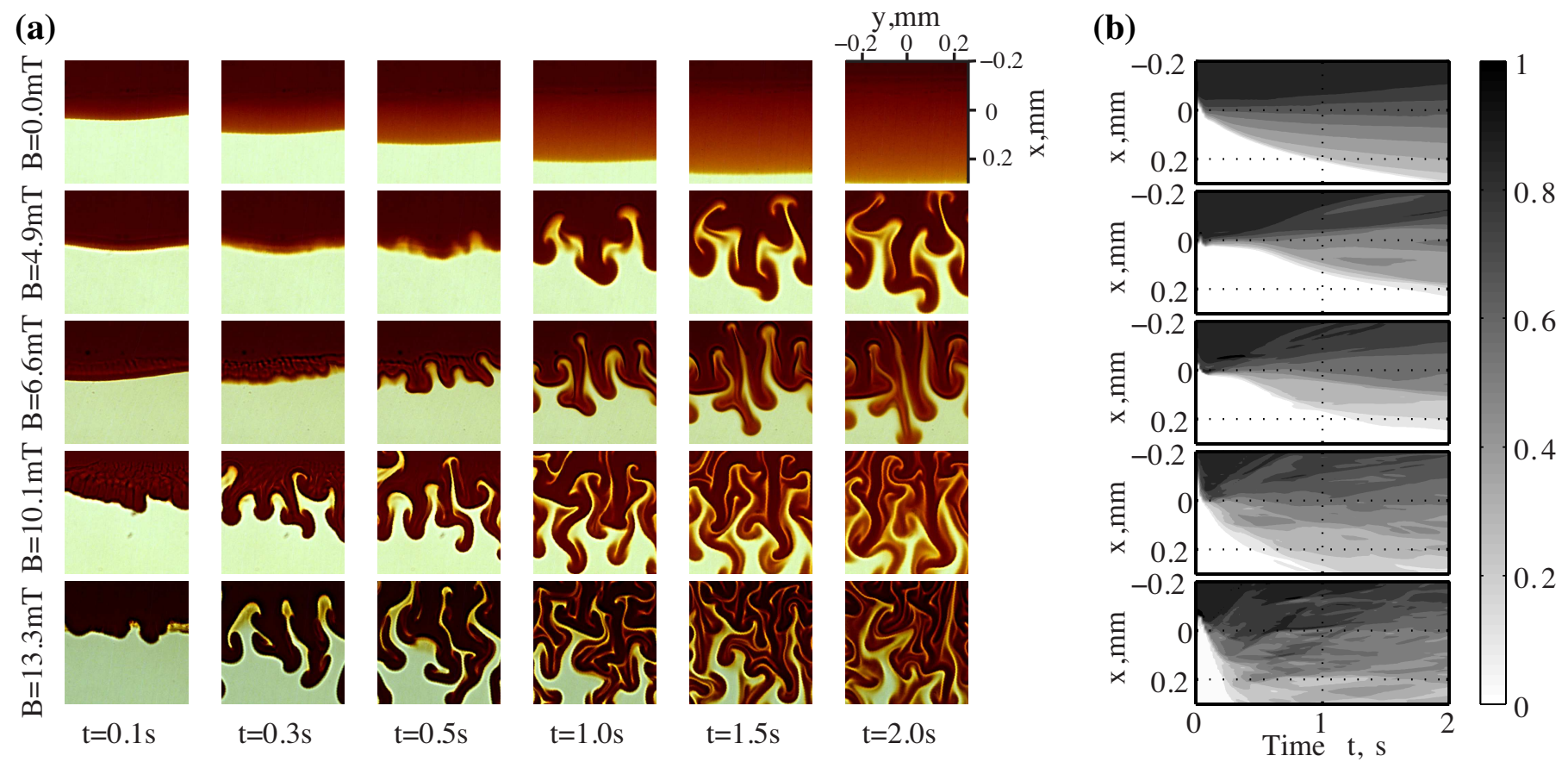

Figure 3: (a) Snapshots of the magnetic micro-convection development at several times for various magnetic fields. The field of view is $0.5 \times 0.5 \mathrm{~mm}^{2}$. (b) Spatially averaged concentration time dependence for the same magnetic fields as in (a) qualitatively revealing mixing enhancement and dynamics.

The experiments are performed for various magnetic field values. Snapshots of magnetic micro-convection development at several time moments can be seen in Fig 3 (a). Larger field provokes a faster evolution of the instability, enhancing mixing. It becomes more apparent, when one observes the spatially averaged concentration $\bar{c}_{t}$ dynamics, shown in Fig 3 (b) contour plots. In the case of diffusion $(B=0)$, more than 2 seconds are needed for mixing to change the initial concentrations $c_{0}$ near the edges of the x-axis $0.5 \mathrm{~mm}$ field of view, whereas for the largest field $B=13.3 \mathrm{mT}$ it happens in less than 0.5 s. Clearly, an increase in the field strength increases the mixing development, although, interface formation influence makes it less notable. 

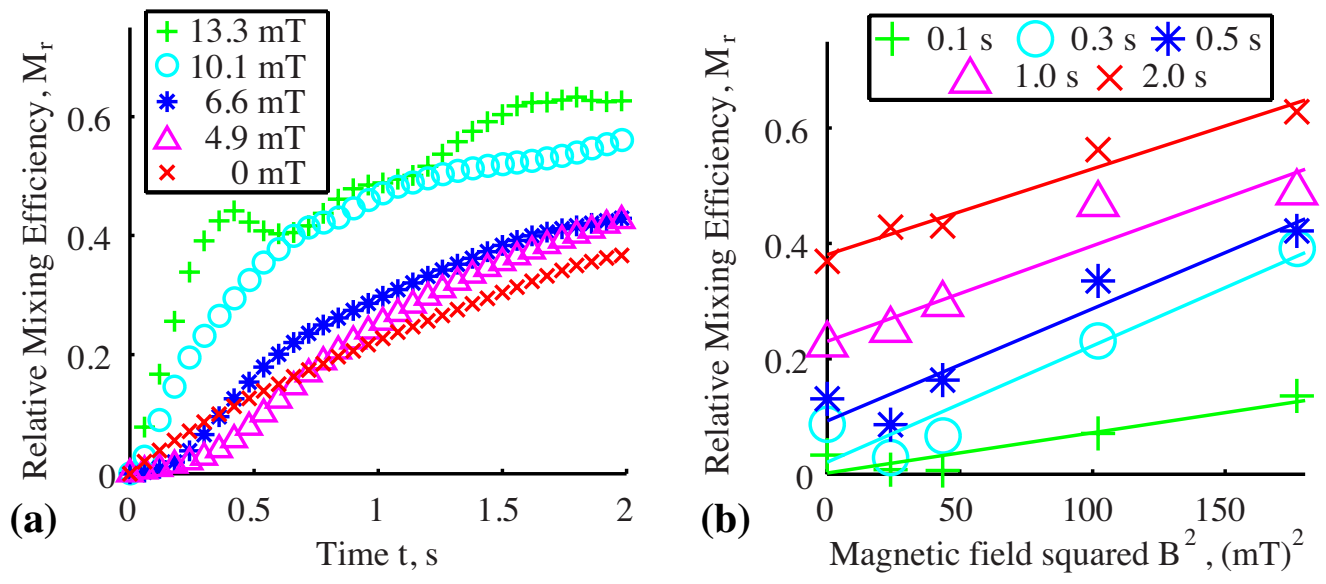

Figure 4: (a) Relative mixing efficiency as a function of time for various magnetic fields. A clear mixing enhancement is visible. (b) Relative mixing efficiency as a function of magnetic field squared for various time moments.

A more quantitative result of the magnetic micro-convection influence can be seen in Fig 4 (a), where relative mixing efficiency $M_{r}(t)$ is showed for the magnetic field values. Achieved $M_{r}$ values might seem small, but it is important to remember its definition (Eq3 34 ) and the experimental cell, which is much larger than the considered field of view $\left(0.5 \times 0.5 \mathrm{~mm}^{2}\right)$ and accordingly has two large basins of the original concentrations, making it very long to reach completely mixed state. Overall, small fields $(B<7 \mathrm{mT})$ seem to enhance mixing over diffusion only slightly, while further increase of magnetic field boosts mixing. This can be explained by Fig 4 (b), where relative mixing efficiency is plotted as a function of magnetic field squared for several time values. Data points in graph agree well with the fitted lines, implying mixing efficiency to be square dependent on the field. This result is consistent with previous findings of the field dependence of magnetic micro-convection characteristics $[6$.

Magnetic field influence on mixing can be analyzed by observing the change in slopes of the fitted lines in Fig 4 (b). The slopes are 0.7, 2.0, 2.0, 1.7 and $1.5 \times 10^{-3} \mathrm{mT}^{-2}$ for $0.1,0.3,0.5,1.0$ and $2.0 \mathrm{~s}$ time values respectively. The variation in slopes for different times arises from the phenomenon and diffusion interplay. At small, but finite times $(t=0.1 \mathrm{~s}, t=0.3 \mathrm{~s})$, the instability forms and enhances mixing, increasing the slope until a maximum. Afterwards $(t>0.5 \mathrm{~s})$, a slow decrease of the slope can be seen, as the concentrations tend to equate. In addition, these fits offer an estimate of the relative mixing efficiency after a selected time as a function of magnetic field. For example, after $t=0.5 \mathrm{~s}$ (linear fit for data marked with asterisk in Fig 4 (b)):

$$
M_{r}(t=0.5 \mathrm{~s})=0.002 \times(\mathrm{B}[\mathrm{mT}])^{2}+0.092,
$$

which is valid for this particular system with $B<20 \mathrm{mT}$. 
A direct comparison between the magnetic micro-convection and other active mixing methods for magnetic/non-magnetic fluid systems is difficult due to the different geometries and fluids used. Wen et. al 3, where mixing in a microchannel is achieved with an AC magnetic field, has mentioned that the characteristic fingers reach both channel walls, which are $\Delta x=0.15 \mathrm{~mm}$ apart, after $t=0.5 \mathrm{~s}$ at $B=14.6 \mathrm{mT}$. In comparison, after the same time and a similar field strength, we have observed that fingers created by micro-convection are $\Delta x=0.5 \mathrm{~mm}$ long, which is 3 times more, although the particle concentration and the initial susceptibility for our magnetic fluid is smaller. Partly this comes from the differences in thicknesses. In Zhu et al. 4 mixing with an in-plane DC field is achieved in a reservoir with a $0.5 \mathrm{~mm}$ radius. The mixing efficiency in this paper is insufficiently explained, allowing us only to surmise that magnetic micro-convection creates instabilities in similar length and time scales for alike magnetic fields.

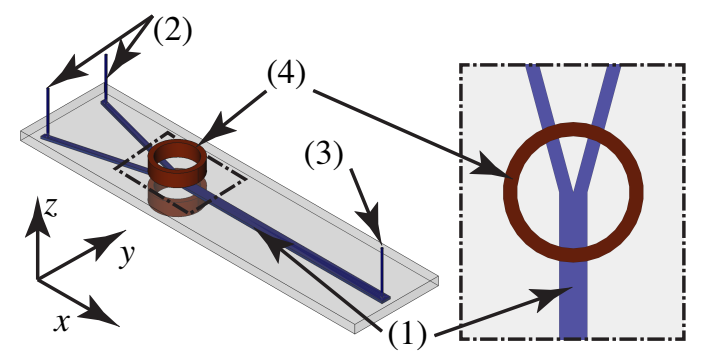

Figure 5: A proposed test setup with microfluidics channel (1), inlets (2), outlet (3) and small coils (4)

The results showed above clearly indicate the advantages of using magnetic micro-convection for mixing. It is very simple to realize it in an already existing microfluidics setup, by adding small coils in the desired place of mixing, as proposed in Fig 5 .

To test this concept, we have made a preliminary experiment in a simple continuous flow microfluidics setup as similar as possible to the proposed concept. The flow cell is made of a ' $Y$ ' shape cut in a single Parafilm $M($ layer $\left(20 \times 50 \mathrm{~mm}^{2}\right)$ with two inlet channels $\left(\approx 5 \times 20 \mathrm{~mm}^{2}\right)$ with $\mathrm{a} \approx 15^{\circ}$ angle between them that join in a common outlet channel $\left(\approx 10 \times 20 \mathrm{~mm}^{2}\right)$. Two glass slides are welded together with this layer as described previously in the section 2. making a flow cell with a $0.12 \mathrm{~mm}$ thickness. The upper glass slide has glued metal tubing connections for inlets (magnetic fluid and water) and outlet. Syringe pump produces a $v=0.15 \mathrm{~mm} / \mathrm{s}$ flow in $x$ direction over the field of view, as determined with a micro Particle Image Velocimetry (Dantec) setup.

Magnetic micro-convection in the flow cell test (see Fig 6) creates a similar pattern as seen above, most likely inducing a comparable mixing enhancement. 


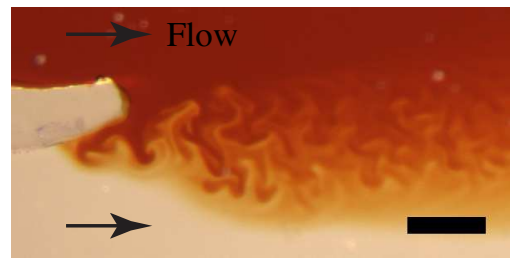

Figure 6: A preliminary test of mixing with magnetic micro-convection in a simple microfluidics flow cell. Scale bar is $0.5 \mathrm{~mm}$.

A more detailed study in a specially designed microfluidics channels should be performed to characterize the applicability of the phenomenon in specific systems, but the concept and its benefit has been showed.

In these experiments the magnetic field is created with a coil system, because it can be easily installed on a microscope stage, without blocking the observation ability of the microscope. Besides, the field strength is adjustable with a power supply and does not involve any moving parts on the microscope stage. However, a homogeneous magnetic field needed for evoking magnetic microconvection can also be obtained differently. For example, permanent magnets can be advantageous in applications where a connection to a power supply is impossible and a flexible adjustment of the used field is not needed. In addition, they can provide high fields while having a small size. Another apparent method is micro-fabrication, where micro-coil or permanent magnet systems can be implemented in the microfluidics chip during its production. In fact, the magnetic field source should be selected after considering the requirements of the desired application and the critical field [6] needed for the magnetic micro-convection.

A limitation of boosting mixing by increasing the magnetic field strength comes from the saturation of magnetic fluid particle magnetization. It is efficient to increase the field only during the linear regime, which can be found from the magnetic fluid magnetization curve and is $B_{\text {sat }} \approx 20 \mathrm{mT}$ for this particular magnetic fluid, as mentioned before (magnetization curve is not showed).

Magnetic nanoparticles are well known in many scientific and clinical applications [10. As magnetic fluid is a colloid that consists of magnetic nanoparticles, it should be possible to realize magnetic micro-convection with any magnetic particle ensemble, if colloidal, magnetic and setup properties are properly combined. For example, we can consider a colloidal dispersion of magnetic particles that is functionalized with antibodies. Mixing it with an analyte in a microfluidics flow cell by using the magnetic micro-convection should decrease the time needed for cell or biomolecule magnetic labeling. Together with a consecutive magnetic sorting and subsequent analysis, an accelerated immunoassay test in a lab on a chip system can be achieved. Other possible applications are in speeding up sorting mechanisms based on ferrohydrodynamics [1] or increasing the mixing speed of selected tracers that are suspended in the magnetic fluid. 


\section{Conclusions}

We have shown that the magnetic micro-convection is a convenient method to enhance mixing in microfluidics systems that use magnetic particle colloids. Comparing to other methods proposed in literature, it is better or of similar efficiency. In addition, it is easy to implement due to the simple requirements of a homogeneous magnetic field in the place wanted. The experiments show the capability to achieve $M_{r} \approx 45 \%$ over a large $0.5 \mathrm{~mm}$ distance in 0.4 seconds with a magnetic field $B<15 \mathrm{mT}$, which is 4 times more and 10 times faster than with diffusion. Mixing efficiency quickly grows with an increasing magnetic field, following a square law, but it is limited by the magnetization saturation. A simple microfluidics test measurement proves the concept. But further measurements in a specially designed microfluidics setup are necessary for full characterization of the magnetic microconvection usage capabilities, including determining the influence of channel size, flow-rate and colloidal properties and reducing the interface formation effect.

\section{Acknowledgements}

This research is supported by a European Social Fund project Nr.2013/0028/1DP/ 1.1.1.2.0/13/APIA/VIAA/054

\section{References}

[1] Y. K. Suh and S. Kang, "A Review on Mixing in Microfluidics," Micromachines, vol. 1, no. 3, pp. 82-111, Sep. 2010.

[2] V. Hessel, H. Löwe, and F. Schönfeld, "Micromixers - a review on passive and active mixing principles," Chemical Engineering Science, vol. 60, no. 8-9, pp. 2479-2501, Apr. 2005.

[3] C.-Y. Wen, C.-P. Yeh, C.-H. Tsai, and L.-M. Fu, "Rapid magnetic microfluidic mixer utilizing AC electromagnetic field." Electrophoresis, vol. 30, no. 24, pp. 4179-86, Dec. 2009.

[4] G.-P. Zhu and N.-T. Nguyen, "Rapid magnetofluidic mixing in a uniform magnetic field." Lab on a chip, vol. 12, no. 22, pp. 4772-80, Dec. 2012.

[5] K. Erglis, A. Tatulcenkov, G. Kitenbergs, O. Petrichenko, F. G. Ergin, B. B. Watz, and A. Cebers, "Magnetic field driven micro-convection in the Hele-Shaw cell," Journal of Fluid Mechanics, vol. 714, pp. 612-633, Jan. 2013.

[6] A. Tatulcenkovs, G. Kitenbergs, K. Erglis, O. Petrichenko, R. Perzynski, and A. Cebers, "Magnetic field driven micro-convection in the Hele-Shaw cell: Brinkman model and its comparison with experiment," 2014, submitted to Physics of Fluids. 
[7] M. M. Maiorov and A. Cebers, "Magnetic microconvection on the diffusion front of ferroparticles," Magnetohydrodynamics, vol. 19, pp. 376-380, 1983.

[8] R.Massart, "Preparation of aqueous magnetic liquids in alkaline and acidic media," IEEE, vol. 17, pp. 1247-1248, 1981.

[9] T. J. Johnson, D. Ross, and L. E. Locascio, "Rapid microfluidic mixing." Analytical chemistry, vol. 74, no. 1, pp. 45-51, Jan. 2002.

[10] A. Ito, M. Shinkai, H. Honda, and T. Kobayashi, "Medical application of functionalized magnetic nanoparticles," Journal of Bioscience and Bioengineering, vol. 100, no. 1, pp. 1 - 11, 2005.

[11] T. Zhu, R. Cheng, S. Lee, E. Rajaraman, M. Eiteman, T. Querec, E. Unger, and L. Mao, "Continuous-flow ferrohydrodynamic sorting of particles and cells in microfluidic devices," Microfluidics and Nanofluidics, vol. 13, no. 4, pp. 645-654, 2012. 